\title{
Patient Characteristics and Inequalities in Doctors' Diagnostic and Management Strategies relating to CHD: A Video-simulation Experiment
}

\author{
Sara Arber* a, John McKinlay ${ }^{b}$, Ann Adams ${ }^{c}$, Lisa Marceau ${ }^{b}$, \\ Carol Link $^{b}$ and Amy O'Donnell ${ }^{b}$
}

a. Centre for Research on Ageing and Gender,

Department of Sociology,

University of Surrey,

Guildford, Surrey GU2 7XH, UK

b. New England Research Institutes,

Watertown, Mass 02172, USA

c. Centre for Primary Health Care Studies,

University of Warwick,

Coventry CV4 7AL, UK

* Corresponding author. Tel: +44-(0)1483-686973;

Fax: +44-(0)1483-689551; e-mail: $\underline{\text { S.Arber@ } \text { surrey.ac.uk }}$

Published in: Social Science and Medicine, 2006, 62 (1): 103-115. 


\begin{abstract}
Numerous studies examine inequalities in health by gender, age, class and race, but few address the actions of primary care doctors. This factorial experiment examined how four patient characteristics impact on primary care doctors' decisions regarding coronary heart disease (CHD).
\end{abstract}

Primary care doctors viewed a video-vignette of a scripted consultation where the patient presented with standardised symptoms of CHD. Videotapes were identical apart from varying patients' gender, age (55 versus 75 ), class and race, thereby removing any confounding from social context of the consultation or other aspects of patients' symptomatology or behaviour. A probability sample of 256 primary care doctors in the UK and US viewed these video-vignettes in a randomised experimental design.

Gender of patient significantly influenced doctors' diagnostic and management activities. However, there was no influence of social class or race, and no evidence of ageism in doctors' behaviour. Women were asked fewer questions, received fewer examinations and had fewer diagnostic tests ordered for CHD. 'Gendered ageism' was suggested, since midlife women were asked fewest questions and prescribed least medication appropriate for CHD. Primary care doctors' behaviour differed significantly by patients' gender, suggesting doctors' actions may contribute to gender inequalities in health.

\title{
Keywords
}

Gender, Ageism, Health Inequalities, Primary care, Decision-making, UK/US, Randomised experiment 


\section{Introduction}

Sociological and epidemiological research on inequalities in health by gender, age, social class and race has largely neglected the actions of health care providers. The dominant paradigm emphasises patient characteristics, including socio-economic status, family background, level of deprivation, working conditions, social support, psycho-social characteristics, lifestyle risk factors and social capital (Bartley, Sacker, Firth \& Fitzpatrick, 1999; Macintyre, 1997). Researchers less often consider supplyside factors associated with doctors' actions as potential sources of health inequalities, e.g. recent books on health inequalities pay scant attention to supply-side factors (cf. Bartley, Blane \& Davey Smith, 1998; Graham, 2000; Mackenbach \& Bakker, 2002).

The supply-side factor addressed here is whether there is differential 'processing of patients' by primary care providers according to patients' social characteristics. McKinlay (1975) examined how different types of organisations process people. There has been more research on processing patients within hospital than primary care. This paper employs a factorial experiment to examine whether four patient characteristics - gender, age, social class and race - influence diagnostic and management decisions of primary care doctors in the UK and US when standardised symptoms of coronary heart disease (CHD) are presented.

Primary care doctors are 'gatekeepers' to secondary care (Forrest, 2003); their initial decisions determine how patients are subsequently processed through the healthcare system, including their investigations and treatment. If patients' social characteristics influence how primary care doctors diagnose and manage patients, specific groups of patients may be disadvantaged in treatments received in primary care and/or less likely to be referred for secondary care. We support Paterson and Judge's statement that 'inequalities in access to secondary care may originate in, and therefore need to be addressed in, the primary care sector' (2002: 170).

Primary care physicians are increasingly acting as gatekeepers to specialists and other medical resources in the US (Forrest, 2003), while UK general practitioners have traditionally performed this role. The UK and US represent contrasting healthcare systems with different payment and funding mechanisms, organisational structures and systems of medical education. The paper uses pooled data from primary care 
practitioners in US and UK, examining whether patients' social characteristics have comparable effects on doctors' behaviour in these two countries.

\section{Socio-demographic inequalities in CHD}

CHD provides an exemplar condition for this study of how primary care doctors process patients. It is the main cause of death for both women and men (Lawlor, Ebrahim \& Davey-Smith, 2002a; Wenger 1997), and commonly presented by midlife and older patients to primary care doctors in the US and UK. The varying rates of CHD by age, gender, social class and race might suggest that primary care doctors' decisions would be influenced by knowledge of these risk profiles (base rates) by patient characteristics.

Rates of CHD increase with advancing age, with age-specific CHD mortality higher among men than women (Lawlor, Ebrahim \& Davey-Smith, 2002b). However, twice as many women as men aged 45-64 have undetected or 'silent' myocardial infarctions, suggesting later CHD diagnosis among women (McKinlay 1996). Women with CHD delay longer before reaching hospital and present with more severe infarcts (Jackson, 1994), possibly reflecting lay beliefs about CHD as a primarily male disease (Emslie, Hunt \& Watt, 2001). CHD mortality has declined in most developed countries over recent years, with greater declines for men than women (Peltonen, Lundberg, Huhtasaari \& Asplund, 2000). Women have poorer prognosis than men following acute myocardial infarction, after adjusting for clinical covariates (Marrugat, Gil \& Sala, 1999). These studies suggest a need for research on health care received by women first presenting with CHD symptoms.

Lower socio-economic groups experience higher rates of $\mathrm{CHD}$, with greater falls in rates among higher than lower social classes (Barnett, Armstrong \& Cooper, 1999; Davey Smith, Hart, Watt, Hole \& Hawthorne, 1998; Kaplan \& Keil, 1993; Lawlor et al. 2002a). Blacks experience higher rates of CHD than whites, resulting in a growing racial divide in CHD mortality (Barnett et al. 1999). Higher levels of cardio-vascular disease indicators among older US blacks are not explained by their poorer socioeconomic status (Rooks, Simonsick, Miles, Newman, Kritchevsky, Schulz \& Harris, 2002). 


\section{Processing patients with CHD in hospital care}

Extensive research has shown differential CHD treatment of women and men within secondary care. Raine's (2000) systematic review of CHD found women are less likely than men to undergo non-invasive diagnostic investigations and receive less surgical treatment. In the US and UK, significantly fewer women than men undergo coronary angiography or bypass surgery (Ayanian \& Epstein, 1991; Dudley, Bowling, Bond, McKee, Scott, Banning, Elder, Martin \& Blackman, 2002; McKinlay, 1996; Sharp, 1994; Sharp, 1998; Shaw, Maxwell, Rees, Ho, Oliver, Ben-Shlomo \& Ebrahim, 2004). Beery's (1995) theoretical discussion of gender bias in CHD-related referrals for diagnostic and therapeutic procedures suggests that gender stereotypes, such as men viewed as more stoical and only likely to complain when really sick, influence doctors' management decisions. The American Medical Association's Council on Ethical and Judicial Affairs (CEJA, 1991) state that physicians need to look for hidden cultural or social bias in their clinical decisions.

Research on gender differences in CHD treatment primarily uses an inequalities framework, whereas research on age differences in treatment is largely characterised as rationing - whether older patients receive lower quality or quantity of health care. The UK government has come out against ageism in the National Service Framework $(N S F)$ for Older People, where Standard 1 is targeted at 'rooting out age discrimination' (DoH, 2001). Although, this rules out using age as an explicit rationing criterion, age may still implicitly influence doctors' decisions about diagnostic testing and referral (Locock, 2000).

It is important to integrate studies of age-related rationing with research on gender differences in CHD diagnosis and treatment. Doctors may vary their diagnostic procedures or treatments in relation to the interaction of patients' age and gender. Among patients admitted with acute myocardial infarction, Shaw et al. (2004) found lower levels of coronary artery bypass grafts among women, with the gender disadvantage in revascularisation rates greater above 75 than at ages 40-64. They note that 'few studies have considered age and gender inequities in conjunction'.

Research has shown lower rates of surgical procedures for CHD among lower socioeconomic groups and blacks compared with whites in the US (Kaplan \& Keil, 1993). 
Similarly Finnish blue collar workers have lower rates of coronary bypass operations than white collar, despite CHD mortality being twice as high amongst the former (Keskimaki, Koskinen \& Salinto, 1997). Hetemaa, Keskimaki, Manderbacka, Leyland $\&$ Koskinen suggest that socioeconomic differences in coronary surgical rates could be caused "by physicians' socially biased referral decisions" (2003: 184). Despite extensive research on bias in medical decision-making, scant attention has addressed patient characteristics (Bornstein \& Emler, 2002).

\section{Processing CHD patients in primary care}

The above studies have examined hospital treatment, Raine (2001: 400) states 'Primary care physicians act as gatekeepers to specialist health services, yet this critical role in the healthcare system has been largely ignored by researchers in this field.' The few available studies have used practice-based data or surveys. Ecological analyses of hospital procedures have shown lower rates of angiography and revascularisation in practices with high deprivation scores (Hippsley-Cox \& Pringle, 2000). Practice-based data show that men with heart disease are more likely to receive lipid-lowering drugs than women, with a greater gender bias among the 45-54 than older age groups (Hippisley-Cox, Pringle, Crown, Meal \& Wynn, 2001), while DeWilde, Carey, Bremner, Richards, Hilton \& Cook (2003) found lower prescription of lipid lowering drugs with increased age, but no sex difference after adjustment for disease severity. A population-based survey in Boston of patients seeking care for heart symptoms found lower cardiologist referral rates among blacks, and white women received less CHD-related treatment than men (Crawford, McGraw, Smith, McKinlay \& Pierson, 1994; McKinlay, 1996).

Analyses of practice databases and cross-sectional surveys primarily focus on prescribing or referrals, rather than the full range of actions of primary care doctors. While they indicate possible gender and age bias, they cannot assess whether patient characteristics per se influence doctors' decisions, because it is impossible to control adequately for differences in symptomology or patients' manner of presentation in the consultation (Raine, 2001). Patients from different social groups may express themselves in varying ways, be more or less assertive, or offer different types of information during the consultation. Thus, despite statistical controls for potentially confounding variables, the possibility remains that findings of these studies reflect 
other uncontrolled differences between patients. As Hippisley-Cox (2004: 412) observes these studies 'do not tell us why inequalities arise or at what point in the total care pathway they are most likely to occur.'

Our research takes a different approach to these methodological difficulties. It addresses whether inequalities occur within the primary care pathway, by designing an experiment which involved showing doctors videotapes of a scripted consultation in which patients presented with CHD symptoms in a standardised way. The videotapes were identical apart from varying patients' gender, age (55 versus 75 ), class and race, and therefore removed any confounding from the social context of the consultation or other aspects of patients' symptomatology or behaviour.

\section{Aims}

This paper aims to examine:

1. To what extent four patient characteristics - gender, age, class and race (singly and in combination) - influence primary care doctors' diagnostic and management decisions for patients presenting with identical symptoms of CHD?

2. Whether there are significant differences between the UK and US in the influence of patient characteristics on primary care doctors' diagnostic and management decisions?

3. Given the risk profiles for CHD by gender, age, class and race, do doctors' diagnostic and management decisions vary in expected directions with these known base rates?

To achieve these aims, we examine the range of actions undertaken by doctors during consultations, each of which can potentially be influenced by patients' social characteristics. Doctors undertake three information gathering activities to assist in diagnosis:- asking the patient additional questions, undertaking a physical examination, and ordering diagnostic tests (Figure 1). They consider the patient's potential diagnoses and estimate their certainty. Once the doctor has a set of preliminary diagnoses, doctors make four management or treatment decisions:- type of prescription given (if any), giving lifestyle or behavioural advice, referral to a specialist, and timing of follow-up visit (Figure 2). Each of these information 
gathering and management decisions potentially influences the way patients are further processed within the healthcare system.

\section{Methods}

A $2^{4}$ experimental design (Cochran \& Cox, 1957) was conducted simultaneously in the UK and US to estimate the unconfounded effects of patient characteristics on doctors' diagnostic and management decisions of patients presenting in a standardised way. 'Patients' in the video-vignette presented with seven signs and symptoms strongly suggestive of CHD including chest pressure; pressure worsened with exertion, stress and eating; relief after resting; discomfort for more than three months; pain through the back between the shoulder blades; elevated blood pressure; family history of heart disease. A key non-verbal cue was incorporated, demonstrated by the 'Levine fist' (clenched fist to the sternum). The 'patient' was portrayed as consulting this doctor for the first time.

This randomized experimental design allowed evaluation individually and simultaneously of a large number of factors that may influence doctors' behaviour, achieving optimal statistical power cost-effectively. The research team has considerable experience conducting such studies (cf. Feldman, McKinlay, Potter, Freund, Burns \& Moskowitz, 1997; McKinlay, Potter \& Feldman, 1996).

Professional actors were used to realistically portray medical encounters on videotape in which the 'patient' presented with signs and symptoms of CHD. The scenario was taped repeatedly, systematically varying the patient's age, race, gender and class. The four patient characteristics were dichotomized: age 55 or 75 years; male or female; white or black (African-American-US; Afro-Caribbean-UK); and middle class (school teacher) or working class (cleaner in UK; janitor in US) (Table 1). Class was also expressed by style of dress and appearance.

Prior to videotaping the vignettes, several tape-recorded role play sessions were conducted with medical advisers. From these, case scripts were developed to ensure they represented actual doctor-patient consultations. Vignettes were scripted to run 78 minutes duration, reflecting average face-to-face consultation time with primary care doctors in UK and US. Practising medical advisers from the US and UK were on 
site for the first filming day to ensure clinical accuracy, especially non-verbal cues. All subsequent vignettes were modeled on this 'master'. One actor portrayed both US and UK patients (with appropriate accent), and the middle and working class patient. Thus, eight actors/actresses were required to represent age, gender, and race. Medical advisers viewed the tapes and were unable to identify nationality of actors, confirming that authentic US and UK accents were portrayed.

In the experiment, doctors viewed two video simulations: a patient presenting with CHD symptoms and another with symptoms of depression. These two videos were randomly assigned to each doctor in terms of order of viewing and the simulated patients' characteristics. Doctors viewed the videos in their consulting rooms, and after each video were asked questions about their diagnostic and management actions for that patient. US and UK interviewers received comparable interviewer training and quality control procedures ensured standardisation in probing and all aspects of interviewer behaviour.

\section{Sample of Doctors}

General practitioners were selected from Health Authority lists for two contrasting UK areas: West Midlands, and Surrey/SE London (Sutton, Merton and Wandsworth). In the US, internists and family practitioners were identified through the Massachusetts Medical Society. Sampling frames were stratified according to UK/US, gender, and year of medical graduation, with random sequential selection of doctors undertaken. Screening calls were conducted to identify eligible doctors, that is UK or US trained, practising at least half-time. Doctors were randomly selected until a sample of 256 doctors was obtained - 128 in Massachusetts; 64 in West Midlands and 64 in Surrey/SE London. In-person interviews were conducted between May 2001 and March 2002, with a response rate of $65 \%$ in US and 60\% in UK. Informed consent was obtained for each participating doctor.

\section{Indicators}

After viewing the video simulated consultation, the interviewer said: 'In answering the next few questions, please remember that I would like you to consider this patient in the context of your current practice'. The doctor was then asked the following questions about his/her diagnostic decision-making (Figure 1): 
(a) Additional questions Would you ask the patient any additional questions before you decide what's going on? What? Anything else?,

(b) Physical examination Would you conduct a physical examination? What would you want to examine? Anything else?

(c) Possible diagnoses Please list what you think is going on with this patient? (What are the possibilities?) Anything else? Using a scale of 0 to 100, with 0 indicating total uncertainty and 100 indicating total certainty about a particular condition, how certain are you that this patient has [condition]?

(d) Diagnostic tests Which are the two most important possibilities you would test for? Based on the information presented, would you order any tests if you saw this patient today? In relation to the most important possibility? What tests would you order? Anything else? And in relation to the second most important possibility? What tests would you order? Anything else?

Questions were then asked about their management of the patient (Figure 2):

(a) Prescriptions Based on the information presented in this case, would you prescribe or recommend any medication for this patient today? What would you prescribe or recommend? Anything else? (A prescription appropriate for treatment of CHD was coded where the doctor answered any of: Antihyperlipidemics, Beta-Blockers, Calcium channel blockers, Aspirin or Vasodilating agents).

(b) Advice giving Would you advise the patient about his/her lifestyle or behaviour today? What would you advise? Anything else?

(c) Specialist referral Would you be likely to refer this patient to another health care professional today? To which type of health care professional would you refer them?

(d) Timing of return visit Would you want to see this patient again? How soon would you want to see this patient again? (coded in days)

\section{Coding and Analysis}

For each question, the interviewer recorded verbatim the doctor's full response. Detailed coding frames were developed in consultation with medical advisers in both countries. They were finalised after achieving over $90 \%$ inter-coder reliability between US and UK coders. The final coding was undertaken by one US coder. 
Analysis of variance was used to assess whether four patient characteristics (gender, age, class and race) had statistically significant effects on each of the above indicators. All effects were estimable and orthogonal in the absence of missing data. A complete model was specified (all main effects and interactions), so that the error term used to test all effects was that due to replication of the experiment (128 degrees of freedom). Proportions and count variables were analysed. ANOVA for count variables (e.g. number of questions asked, number of parts of the body examined) used the square root transformation to minimise any effects of outliers (high counts). Precise $p$-values are reported in tables. The main findings discussed in the paper are significant at least at $p<.05$. The paper analyses pooled UK/US data. Tests for interactions with country (UK/US) were conducted and reported where significant at the $\mathrm{p}<.05$ level.

\section{Results}

Lower social classes and blacks are more likely to suffer from CHD than higher social classes and whites, therefore it was surprising that class was not significantly associated with any aspect of doctors' information gathering or the four areas of patient management. There were no significant associations between race and doctors' diagnostic actions, and only one with a management decision, namely higher referral of blacks to specialist cardiology facilities ( $27 \%$ of blacks and $15 \%$ of whites referred). The results for class and race are presented in Appendix Tables 1 and 2. Given the lack of main effects of patients' class and race on doctors' diagnostic and management decisions, analyses by class and race are not considered further in this paper. In contrast, there were several significant associations between primary care doctors' actions and patients' gender and/or age.

\section{Gender of patient}

Gender has a significant influence on all four aspects of doctors' diagnostic strategies; in each case women receive less attention than men presenting with CHD symptoms (Table 2). Doctors would ask men more questions than women (on average 7 and 5.7 questions respectively), and perform more extensive examinations for men than women (5.1 compared to 4.3 parts of the body or body systems would be examined respectively). 
CHD was mentioned as a possible diagnosis for more men than women (95\% and $88 \%$ respectively), although this marginally failed to reach statistical significance $(p=.052)$. Doctors had significantly higher certainty of CHD for male than female patients, $57 \%$ and $47 \%$ respectively, on a scale of 0 (total uncertainty) to $100 \%$ (total certainty), Table 2c.

There was no gender difference in number of tests doctors would order for the two most important possible diagnoses they wished to test for. However, more doctors would order tests for a possible diagnosis of CHD for male than female patients, $90 \%$ and $80 \%$ respectively, with more of these tests ordered for male than female patients, averaging 3 and 2.1 respectively (Table $2 \mathrm{~d}$ ).

Turning to management decisions, doctors would prescribe medication appropriate for treating heart disease at the first consultation to $64 \%$ of male and $52 \%$ of female patients (Table 3a). However, there was no significant gender difference in how many pieces of lifestyle or behavioural advice would be given, referral to a specialist, or recommended timing of next appointment.

Our findings indicate that women presenting with CHD symptoms are disadvantaged in primary care. Doctors provide a less thorough diagnostic search procedure than for men presenting with identical symptoms, and fewer women are given prescriptions appropriate for treating CHD.

\section{Age of patient}

There is mounting concern about whether doctors may unconsciously or consciously alter their diagnostic or management behaviour because of the patient's age. Regarding diagnostic strategies, there was no evidence that doctors asked older (age 75) patients fewer additional questions than midlife (age 55) patients, or that age influenced number of physical examinations the doctor would perform (Table 2). Similarly, there was no significant effect of age on likelihood of making a possible diagnosis of CHD or doctors' certainty of CHD diagnosis. The only diagnostic area where age was linked to doctors' behaviour was number of diagnostic tests. Older patients would be ordered more diagnostic tests than midlife patients (Table $2 \mathrm{~d}$ ). This related both to number of tests ordered for the two possibilities the doctor most wished to test for (average 5.3 tests for 
older and 4.4 for midlife patients), and tests ordered specifically for a possible CHD diagnosis (2.9 and 2.1 tests respectively).

We examined whether doctors' diagnostic decisions varied with patients' age in different ways in the US and UK, finding significant first-order interactions between country (or healthcare system) and age for ordering diagnostic tests (Table 4a). Age influenced test ordering in UK but not US. Older UK patients (age 75) would have more diagnostic tests ordered (on average 6 tests compared to 4.3 for midlife patients), more would have a test ordered for a possible diagnosis of CHD ( $88 \%$ compared to $73 \%$ for 55 year olds), and would have twice as many tests ordered for a possible CHD diagnosis (average of 3.2 tests compared to 1.6 for 55 year olds). These findings are the reverse of ageism, indicating that older patients presenting with CHD symptoms in the UK would have more extensive diagnostic testing to assist accurate diagnosis than midlife patients. There was no evidence that age influenced doctors' test ordering in US.

Turning to management decisions, age of patient was not significantly associated with doctors' prescribing, advice giving or specialist referral (Table 3). However, older patients would have been asked to revisit their doctor sooner than midlife patients, on average 10.2 compared with 12.1 days respectively. Despite no significant main effect of age on referral in the pooled UK/US data, there was a significant interaction between age and country for referral to a cardiologist or specialist coronary facility (Table 4b). In the UK, older ( 75 year old) patients were less likely to be referred than middle aged, $27 \%$ compared with $36 \%$ respectively. This may provide some evidence of possible ageism in UK healthcare treatment for CHD symptoms. In the US, where there were fewer cardiac specialist referrals, the reverse was the case with referrals much higher for older than midlife patients, $16 \%$ and $5 \%$ respectively.

\section{Discrimination against mid-life women}

We showed earlier that women consulting with CHD symptoms received less extensive diagnostic attention from primary care doctors than equivalent men: they were asked fewer questions, had less extensive examinations, and fewer diagnostic tests. Also fewer received CHD appropriate medication. This section examines statistically significant interactions between gender and age of patients, and with healthcare system (UK/US). 
Our findings suggest that midlife women are least likely to be diagnosed and treated aggressively by doctors for $\mathrm{CHD}$, while this occurs most frequently for midlife men.

Doctors would ask midlife men more additional questions (7.9 questions on average), with midlife women asked 5.2 questions (Table 5a). Only $70 \%$ of midlife women would be asked 4 or more additional questions compared with $92 \%$ of midlife men. There was no gender difference among older patients regarding how many additional questions doctors would ask (about 6.2 questions). A significant three-way interaction with country showed that the disadvantage of midlife women in terms of limited questioning occurs in UK, but not US (Table 6). Only $47 \%$ of midlife British women would be asked 4 or more additional questions by their GP, compared with $88 \%$ of midlife men. In the US, over $90 \%$ of female and male patients irrespective of age would be asked 4 or more additional questions. Our findings suggest that UK general practitioners are less likely to activate extensive search procedures in the form of detailed questioning of midlife women presenting with CHD symptoms.

Midlife women are also disadvantaged in two further areas of doctors' diagnostic and management behaviour: certainty of CHD diagnosis and prescribing (but there were no significant interactions with country). Doctors are least certain about a diagnosis of CHD for midlife women (average $41 \%$ certainty) and most certain for midlife men (59\% certainty) (Table 5b). Fewer midlife women are prescribed CHD-related medication than midlife men, $41 \%$ and $66 \%$ respectively (Table 5c). Given that doctors are much less certain that CHD is the 'correct' diagnosis for midlife women, one might expect they would ask midlife women more additional questions to try to 'firm up' their diagnosis, but the reverse was the case, with doctors asking midlife women fewer questions.

\section{Discussion}

There are major methodological difficulties in researching whether primary care doctors' diagnostic and management strategies are influenced by the sociodemographic characteristics of patients. The present study addressed these problems by developing video-vignettes of doctor-patient consultations in which 'patients' (played by professional actors) presented with standardised symptoms of CHD, but varying their gender, age (55 versus 75 ), class and race. A probability sample of 256 
primary care doctors in UK and US were asked how they would diagnose and manage a patient presenting with standardised CHD symptoms in a randomised factorial experiment.

The video-vignette was portrayed as the patient's first consultation. Although, doctors may prefer to enact some of their management decisions (e.g. prescribing or referral) in subsequent consultations, any such preference about timing of diagnostic tests, prescribing medication or making specialist referrals would not be expected to vary with patients' social characteristics.

There was no evidence of patients' class influencing any aspect of primary care doctors' decision-making or treatment. Since the working class and blacks are more likely to suffer from CHD than the middle class or whites, differential processing of patients by class and race may be expected, e.g. higher certainty of CHD. However, our findings suggest that lower class patients and blacks are treated as though their risk profiles and probabilities of CHD are equivalent to those of middle class patients and whites. An issue is therefore whether equivalent treatment, given the known class/race differences in risk factors/disease probabilities, reflects discrimination.

This experiment found no evidence of age discrimination or age-related rationing related to CHD in primary health care. Older patients (age 75) were asked equivalent numbers of additional questions and given equally extensive physical examinations as 55 year olds. Older patients had somewhat more diagnostic tests ordered, and were asked to return sooner to see the doctor. The latter might be expected because of greater co-morbidity among older than midlife patients. There was some evidence of age-related actions of doctors in the UK but not US. Older patients were ordered significantly more diagnostic tests in UK than midlife patients, but fewer were referred to a cardiologist or specialist cardiac facility. This suggests possible ageism relating to cardiology referrals in UK, but not regarding diagnostic testing.

Gender was the main patient characteristic systematically influencing doctors' diagnostic behaviour regarding CHD. Women were asked fewer additional questions, given fewer physical examinations, ordered fewer diagnostic tests, and fewer were prescribed CHD-related medication. These gender differences suggest that women 
presenting in primary care with CHD symptoms may be less likely to receive an accurate diagnosis and appropriate treatment than men. Our research shows that these disadvantages are largely the province of mid-life (55 year old) women, since there is little gender difference in doctors' diagnostic strategies or prescribing among older patients.

The gender stereotyping of CHD as primarily affecting midlife men may explain why midlife men received the greatest attention from doctors. Midlife men are seen as the 'archetypal' coronary victim, while midlife women with equivalent symptoms receive less extensive diagnostic attention and fewer management actions. The term 'gendered ageism' is used where ageism starts earlier for women than men regarding employment and promotions in specific occupations (Bernard, Itzin, Phillipson \& Skucha, 1995). Our findings could be seen as a form of 'gendered-ageism' among primary care practitioners, since the disadvantages faced by women with CHD symptoms are restricted to a certain age group, namely midlife rather than older women. Evidence of gendered ageism with regard to doctors asking midlife women fewer questions occurred in the UK, but not US.

Doctors' decision-making processes may have been influenced by the known lower CHD risk profiles for midlife women than men, resulting in less questioning, examinations and prescribing. However, this contrasts with our findings that patient's class and race did not influence doctor's decision-processes, despite the well-known CHD risk profiles by class and race. It is important to consider whether patient characteristics, such as the combination of age and gender, are legitimate cues for the doctor, as they may also be discriminatory if they trigger stereotypes in doctor's minds that can obscure clinical variation and signs of disease.

Our findings move the focus of health variations work back to the doctor-patient relationship, by suggesting that supply-side factors may contribute to gender inequalities in health. The less extensive diagnostic search procedures used by primary care doctors when midlife women present with CHD symptoms may be problematic if doctors fail to identify potentially important cues from women, resulting in the possibility that they may receive sub-optimal care. Given that doctors had a lower certainty of CHD for midlife women, it might be expected that they would undertake 
more questioning and conduct more examinations for midlife women to help firm up their diagnostic certainty.

\section{Limitations and ways forward}

There is no foolproof way of researching bias in doctors' diagnostic and management procedures related to patients' gender, age, class or race. Alternative methods have varying strengths and weaknesses. They include analysis of practice-based administrative records, such as Hippisley-Cox \& Pringle (2000), Hippisley-Cox et al. (2001) and DeWilde et al. (2003); and patient self-report surveys about consultations, receipt of prescriptions or specialist referrals (e.g. Crawford et al., 1994; McKinlay, 1996). Both these research approaches are important in identifying lower levels of procedures, prescriptions or referrals among patients with specific social characteristics. However, their findings may occur because of varying severity of symptoms, or differences in actions or assertiveness of patients during consultations. For example, patients who are more educated, more knowledgable, or have more sophisticated skills in dealing with bureaucratic organisations, may receive more thorough examination or appropriate referrals.

Another approach is to undertake observational research using videotapes (or audiotapes) of consultations. Such studies have found that middle class patients volunteer more information, have longer consultations, and receive more explanations from GPs (Boulton, Tuckett, Olson \& Williams, 1986; Pendleton \& Bochner, 1980). However, the diversity of reasons for primary care consultations means it is impossible to conduct observational studies of how primary care doctors manage 'real' patients presenting with CHD-symptoms. This contrasts with conducting observational studies in hospitals, where patients consult with cardiology specialists. Within observational studies of 'real' doctor-patient consultations there may remain difficulties of interpreting findings because of variations in severity of conditions, and patients' communication styles. Both of which may be important explanations for any differences in provider-behaviour found in these 'naturally occurring' consultations.

A final approach is to adopt the strategy used in this study, which has the strength of standardising the severity and manner of presentation of symptoms, and all aspects of the patient's verbal and non-verbal behaviour. All doctors were responding to patients 
with identical signs and symptoms of CHD thereby enhancing internal validity; the only aspects that varied were age, gender, class and race.

However, a potential limitation of our approach is that doctors may not respond to simulated patients in the same way as they would to 'real' patients. Use of hypothetical 'patients' potentially threatens external validity (whether a doctors' response to videotaped encounters reflects their usual behaviour in everyday 'real' practice encounters). Our research took four precautionary steps to minimise this potential problem. First, considerable effort was devoted to ensuring clinical realism of the videotaped consultation by using professional actors and filming with experienced clinician advisers present. Second, doctors were specifically asked how typical the videotaped 'patient' was compared with patients they encounter in everyday practice ( $92 \%$ considered them 'very typical' or 'reasonably typical'). Third, doctors viewed the tapes in the context of their practice day (not at a professional meeting, course update or in their home). It was likely they saw real patients before and after viewing the 'patient' in the videotape. Fourth, doctors were specifically instructed to view the 'patient' as one of their own patients and respond as they would typically respond in their own practice.

Most research addressing health inequalities has focused on risk factors and patients' behaviour, rather than actions of healthcare providers. It behoves researchers to use the full armoury of research methodologies to assess whether inequalities in health are associated with the supply-side of healthcare. Large-scale practice databases and surveys can describe the extent and nature of potential supply-side inequalities, whereas qualitative and experimental methods help uncover mechanisms underlying these inequalities. Such a combination of approaches provides the most fruitful basis for devising policies to ameliorate health inequalities. 


\section{Acknowledgements}

Research funded by National Institutes of Health, National Institute of Aging, Grant no. AG-16747. We are grateful to Alan Goroll, MD, Ted Stern, MD, John Stoeckle, MD (Massachusetts General Hospital), David Armstrong, PhD, FFPHM, FRCGP and Mark Ashworth, MRCP, MRCGP (United Medical Schools of Guys, Kings and St Thomas's, London), Diane Ackerley, MBBS (Guildford and Waverley Primary Care Trust), Sue Venn (UK research administrator), Sam Colt and Cathie McColl (interviewers), and Nathan Hughes (data entry). We thank the 256 doctors who participated in this research. 


\section{References}

Ayanian, J.Z. \& Epstein, A.M. (1991). Differences in the use of procedures between women and men hospitalized for coronary heart disease, New England Journal of Medicine, 325, 221-225.

Barnett, E., Armstong, D.L. \& Cooper, H.L. (1999). Evidence of increasing Coronary Heart Disease mortality among Black men of lower social class. Annals of Epidemiology. 9(8), 464-471.

Bartley, M., Blane, D. \& Davey-Smith, G. (Eds.) (1998). The sociology of health inequalities, Oxford: Blackwell.

Bartley, M., Sacker, A., Firth, D. \& Fitzpatrick, P. (1999). Understanding social variation in cardiovascular risk factors of men and women: the advantage of theoretically based measures, Social Science and Medicine, 49, 831-845.

Beery, T.A. (1995). Diagnosis and treatment of cardiac disease. Gender bias in the diagnosis and treatment of coronary artery disease, Heart \& Lung, 24(6), 427-434

Bernard, M., Itzin, C., Phillipson, C. \& Skucha, J. (1995). Gendered work, gendered retirement. In S. Arber. \& J. Ginn (eds.) Connecting Gender and Ageing. Buckingham: Open University Press.

Bornstein, B.H. \& Emler, A.C. (2002) Rationality in medical decision-making: a review of the literature on doctors' decision-making biases, Journal of Evaluation in Clinical Practice, 7(2), 97-107.

Boulton, M., Tuckett, D., Olson, C. \& Williams, A. (1986). Social class and the general practice consultation. Sociology of Health and Illness. 8(4), 325-350.

Cochran, W.G. \& Cox G.D. (1957). Experimental designs, $2^{\text {nd }}$ edn. London: John Wiley and Sons. 
Council on Ethical and Judicial Affairs, American Medical Association (CEJA) (1991). Gender disparities in clinical decision making. Journal of the American Medical Association. 266 (4), 559-562.

Davey-Smith, G., Hart, C., Watt, G., Hole, D. \& Hawthorne, V. (1998). Individual social class, area-based deprivation, cardiovascular disease risk factors, and mortality: the Renfrew and Paisley Study, Journal of Community Health, 52, 399-405

Department of Health (2001). National Service Framework for older people, London: HMSO.

DeWilde, S., Carey, I. M., Bremner, S. A., Richards, N., Hilton, S. R. \& Cook, D.G. (2003). Evolution of statin prescribing 1994-2001: a case of agism but not sexism? Heart, 89, 417-421.

Dudley, N., Bowling, A., Bond, M., McKee, M., Scott, M.M., Banning, A., Elder, A.T., Martin, A.T. \& Blackman, I. (2002). Age- and sex-related bias in the management of heart disease in a district general hospital. Age and Ageing. 31, 37-42.

Emslie, C., Hunt, K. \& Watt, G. (2001) Invisible women? The importance of gender in lay beliefs about heart problems, Sociology of Health and Illness, 23, 203-233.

Feldman, H.A., McKinlay, J.B., Potter, D.A., Freund, K.M., Burns, R.B. \& Moskowitz, M.A. (1997). Non-medical influences on medical decision-making: An experimental technique using videotapes, factorial design, and survey sampling, Health Services Research, 32(3), 343-365.

Forrest, B. (2003) Primary care gatekeeping and referrals: effective filter or failed experiment? British Medical Journal, 326, 692-695.

Graham, H. (ed.) (2000). Understanding health inequalities. Buckingham: Open University Press. 
Hetemaa, T., Keskimaki, I., Manderbacka, K., Leyland, A.H. \& Koskinen, S. (2003). How did the increase in the supply of coronary operations in Finland affect socioeconomic and gender equity in their use, Journal of Epidemiology and Community Health, 57, 178-185.

Hippisley-Cox, J. (2004) Inequalities in access to care for patients with ischaemic heart disease, British Journal of General Practice, 54, 411-412.

Hippisley-Cox, J. \& Pringle, M. (2000) Inequalities in access to coronary angiography and revascularisation: the association of deprivation and location of primary care services, British Journal of General Practice, 50, 449-454.

Hippisley-Cox, J., Pringle, M. Crown, N., Meal, A. \& Wynn, A. (2001) Sex inequalities in ischaemic heart disease in general practice: cross sectional survey, British Medical Journal, 322, 832.

Jackson, G. (1994) Coronary artery disease and women, British Medical Journal, 309, 555-557.

Kaplan, G.A. \& Keil, J.E. (1993). Socioeconomic factors and cardiovascular disease: A review of literature, Circulation, 88(4), 1973-1998.

Keskimaki, I., Koskinen, S., \& Salinto, M. (1997). Socioeconomic and gender inequities in access to coronary artery bypass grafting in Finland, European Journal of Public Health, 7, 392-7.

Lawlor, D.A., Ebrahim, S. \& Davey Smith, G. (2002a). A life course approach to coronary heart disease and stroke. In D. Kuh \& R. Hardy (Eds.) A life course approach to women's health (pp. 86-120). Oxford: Oxford University Press.

Lawlor, D.A., Ebrahim, S. \& Davey Smith, G. (2002b). Role of endogenous oestrogen in aetiology of coronary heart disease: analysis of age related trends in coronary heart disease and breast cancer in England and Wales and Japan, British Medical Journal, $325,311-312$. 
Locock, L (2000). The changing nature of rationing in the UK National Health Service. Public Administration. 78(1), 91-109.

Macintyre, S. (1997). The Black Report and beyond. What are the issues? Social Science and Medicine, 44(6), 723-745.

Mackenbach, J. \& Bakker, M. (Eds.) (2002). Reducing inequalities in health: A European perspective, London: Routledge.

Marrugat, J., Gil, M. \& Sala, J. (1999). Sex differences in survival rates after acute myocardial infarction, Journal of Cardiovascular Risk, 6(2), 89-97.

McKinlay, J (1975). Processing people: Cases in organisational behaviour. Woking: Unwin Brothers

McKinlay, J. (1996). Some contributions from the social system to gender inequalities in heart disease, Journal of Health and Social Behaviour, 37, 1-26.

McKinlay, J.B., Potter, D. \& Feldman, H. (1996). Non-medical influences on medical decision-making, Social Science and Medicine, 42, 769-776.

Paterson, I. \& Judge, K. (2002). Equality of access to health care. In J. Mackenbach \& M. Bakker (Eds.) Reducing inequalities in health: A European perspective (pp 169-187), London: Routledge.

Peltonen, M., Lundberg, V., Huhtasaari, F. \& Asplund, K. (2000). Marked improvement in survival after acute myocardial infarction in middle-aged men but not in women. The Northern Sweden MONICA study 1985-94, Journal of Internal Medicine, 247(5), 57987.

Pendleton, D.A. \& Bochner, S. (1980). The communication of medical information in general practice consultations as a function of patients' social class, Social Science and Medicine, 14A, 669-673. 
Raine, R. (2000). Does gender bias exist in the use of specialist health care? Journal of Health Service Research and Policy, 5(4), 237-249.

Raine, R. (2001) Sex inequalities in ischaemic heart disease in primary care, British Medical Journal, 323, 400.

Rooks, R.N., Simonsick, E.M., Miles, T., Newman, A., Kritchevsky, S.B., Schulz, R. \& Harris, T. (2002). The association of race and socioeconomic status with cardiovascular disease indicators among older adults in the Health, Aging, and Body Composition Study, Journal of Gerontology, 57B (4), S247-S256. - 44 words

Sharp, I. (1994). Coronary heart disease: Are women special? London: National Heart Forum.

Sharp, I. (1998). Gender issues in the prevention and treatment of Coronary Heart Disease. In L. Doyal (Ed.) (pp 100-112) Women and health services: An agenda for change, Buckingham: Open University Press.

Shaw, M., Maxwell, R., Rees, K., Ho., D, Oliver, S., Ben-Shlomo Y. \& Ebrahim, S. (2004) Gender and age inequality in the provision of coronary revascularisation in England in the 1990s: is it getting better? Social Science and Medicine, 59 (12), 24992507.

Wenger. N. K. (1997). Coronary heart disease: an older woman's major health risk', British Medical Journal; 315, 1085-1090. 